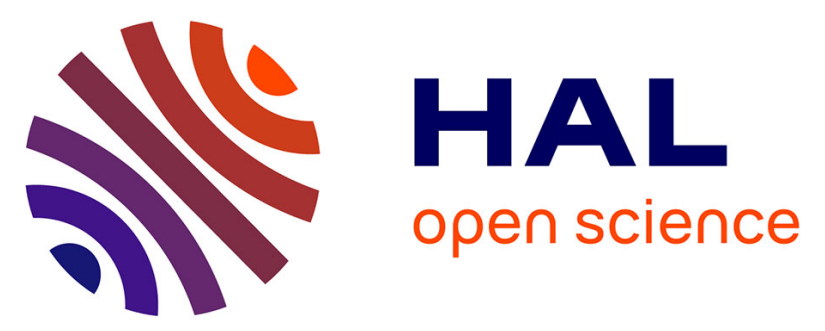

\title{
Third order non-linear optical susceptibilities $(\chi 3)$ of yttria stabilized cubic hafnium(IV) oxide
}

Hassan Denawi, Jacques Demarais, C.S. Praveen, Michel Rérat, Panaghiotis

Karamanis

\section{- To cite this version:}

Hassan Denawi, Jacques Demarais, C.S. Praveen, Michel Rérat, Panaghiotis Karamanis. Third order non-linear optical susceptibilities ( $\chi 3$ ) of yttria stabilized cubic hafnium(IV) oxide. Chemical Physics Letters, 2021, 785, pp.139157. 10.1016/j.cplett.2021.139157 . hal-03418749

\section{HAL Id: hal-03418749}

https: / hal-univ-pau.archives-ouvertes.fr/hal-03418749

Submitted on 26 Nov 2021

HAL is a multi-disciplinary open access archive for the deposit and dissemination of scientific research documents, whether they are published or not. The documents may come from teaching and research institutions in France or abroad, or from public or private research centers.
L'archive ouverte pluridisciplinaire HAL, est destinée au dépôt et à la diffusion de documents scientifiques de niveau recherche, publiés ou non, émanant des établissements d'enseignement et de recherche français ou étrangers, des laboratoires publics ou privés. 
3 Hassan Denawi ${ }^{1}$, Jacques K. Demarais ${ }^{1,2}$, C.S. Praveen ${ }^{3}$, Michel Rerat ${ }^{1}$ and

4 PANAGHIOTIS KARAMANIS ${ }^{1 *}$

$5 \quad{ }^{I}$ E2S UPPA, CNRS, IPREM, Université de Pau et des Pays de l Adour, 64053 Pau, France

$6 \quad{ }^{2}$ International School of Photonics (ISP), Cochin University of Science and Technology

7 (CUSAT), Kalamassery,Ernakulam, Cochin, Kerala, 682022, India

$8{ }^{3}$ Dipartimento di Chimica, Università di Torino, via Giuria 5, 10125 Torino, Italy blications Department

*panagiotis.karamanis@gmail.com

\begin{abstract}
This work reports for the first time theoretical third order nonlinear optical susceptibilities of YSH bulk phases which are key properties for telecommunication technologies and integrated photonic devices. Our outcomes, relying on coupled perturbed Kohn-Sham density functional theory expose that the NLO capacity of YSH should be similar to another material of this family, namely, yttria stabilized zirconia that has been proposed as a promising candidate for integrated photonics by Marcaud et al [Photon. Res. 8, 110 (2020)]. Finally, evidence implying important vibrational contributions to the optical nonlinearities of the systems are revealed and analyzed.
\end{abstract}

\title{
1. Introduction
}

The key to harness light's nonlinear effects and to unlock its "limitless" potential in telecommunication technologies lies in the successful and cost-effective fabrication of fully operating integrated nonlinear photonic devices. [1,2] Upon success, ultra-fast optical processing at femtosecond time scales, having the capacity to give access to petahertz telecommunication bandwidths, could eventually be achieved. In this realm, one of the main properties of interest is the so called intrinsic third order non-linear optical (NLO) susceptibility, 3] routinely designated as $\chi^{(3)}$. The latter band-gap dependent property provides the means to control light with light, [4] via the so-called Kerr effect which reflects the ability of a given material to change its refractive index upon the application of an electric field. In this context, Marcaud et al. [5] reported the outcomes of a joint experimental and theoretical work dealing with the NLO behavior of yttria stabilized zirconia (YSZ). As demonstrated in that work, YSZ, that can be grown epitaxially[6] on silicon substrates, features a comparable third order 
32

33

34

35

36

37

38

39

40

41

42

43

44

45

46

47

48

49

50

51

52

53

nonlinear effective Kerr coefficient $\left(n_{2}\right)$ with silicon nitride.[7] The latter material although it is one of the materials of choice for integrated photonics it suffers from significant shortcomings due to strong two photon absorption (TPA) effects that induce significant optical losses (see ref [5] and references therein). As Marcaud et al. concluded, TPA loses in YSZ are expected to be negligible thanks to its large bandgap and its quasi-cubic local atomistic structure. [5]

Motivated by the intense technological interest in the optical properties of functional oxides for integrated photonic devices worldwide, in this article, we report on reliable $\chi^{(3)}$ estimations of another widely recognized functional oxide, namely, yttria stabilized hafnia (YSH). Hafnia $\left(\mathrm{HfO}_{2}\right),[8]$ the parent material of $\mathrm{YSH}$, is known to possess four crystalline phases[9], namely, tetragonal, monoclinic, cubic and amorphous. Out of the four hafnia phases the third one (c$\mathrm{HfO}_{2}$ ) is of great interest in CMOS technologies owed to its high dielectric constant and wider band gap. [10] However, cubic hafnia becomes "functional" only in quite high temperatures $\left(>2500{ }^{\circ} \mathrm{C}\right)[11]$ and this impedes its application in many areas including optoelectronics. Therefore, its stabilization at ambient conditions via doping is one of the preferred options. For this task, $\mathrm{Y}_{2} \mathrm{O}_{3}$ has proven a highly effective doping agent since it "stabilizes" hafnia's cubic phase even in relatively low concentrations ranging from $0.6 \mathrm{~mol} \%$ to $6.5 \mathrm{~mol} \%$. In particular, the current work reports on accurate coupled perturbed electronic static $\chi_{i i i i, i i j j}^{(3)}$ macroscopic data defined as:

$$
\chi_{i i i i, i i j j}^{(3)}=2 \pi \gamma_{i i i i, i i j j}^{e}(3 V)^{-1} \text { (where, } \mathrm{i} \text { and } \mathrm{j} \text { are the cartesian cell axes) }
$$

In Eq. (1) $\gamma^{e}$ is electronic contribution of the second hyperpolarizability per unit cell volume (V). The latter quantity can be determined experimentally ${ }^{7}$ via measurements of the refractive 
54 index: $\hat{n}_{2}=3 \hat{\chi}^{(3)}\left(4 c \epsilon_{0} \epsilon\right)^{-1}\left(\epsilon\right.$ is the relative dielectric in-plane $\epsilon_{x x}$ component, $\epsilon_{0}$ the

55 electric permittivity of vacuum and $\mathrm{c}$ the light velocity and $\hat{\chi}^{(3)}$ is the effective third order 56 susceptibility[5]).

57

58

59

60

61

62

63

64

65

66

67

68

69

70

71

\section{Results and discussion}
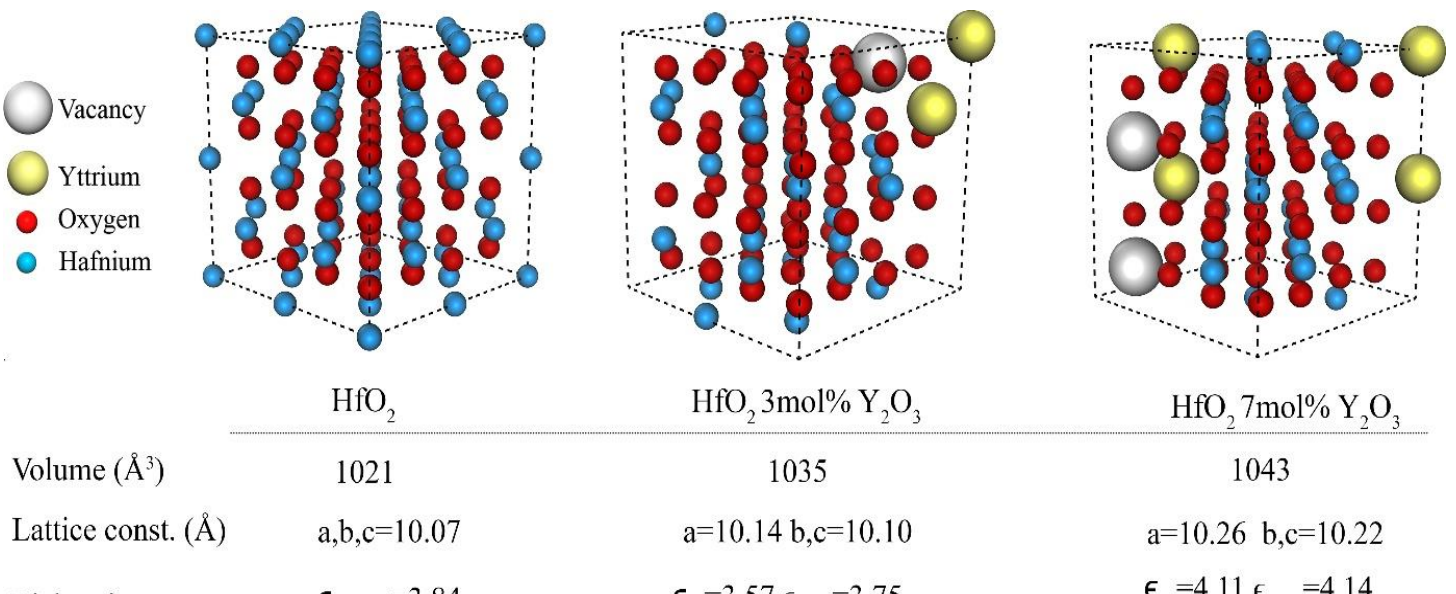

Lattice const. $(\AA)$

Dielectric components $\quad \epsilon_{x x, y y, z z}=3.84$

Fig. 1. Relaxed stoichiometric atomistic super-cells YSH. All structures are true minima of the corresponding supercell potential energy super-surfaces.

All computations presented and discussed here have been carried out using widely tested density functional methods (DFT) as implemented in CRYSTAL17 code. [11] In brief, the generalized gradient approximation (GGA) functional of Perdew, Burke, and Ernzerhof (PBE) and the hybrid functionals PBE0 and B3LYP, have been employed to check the method dependence on the properties of interest. A supercell size of $2 \times 2 \times 2$ has been considered for the disordered and vacancy calculations with a corresponding Monkhorst-Pack grid [12] of $8 \times$ $8 \times 8$. The spatial doping/vacancy distribution chosen has been based on earlier benchmark computations initially carried out on YSZ [5] and applied here on YSH. Although our structural investigation is not exhaustive, our experience on YSZ [5] phases suggests that there is a minor influence of the local atomistic structures of such oxides on the electronic part of their third 
72 order nonlinearities which is expected to dominate the overall response. The computation of 73 the linear and nonlinear properties addressed here relied on coupled perturbed Kohn-Sham 74 density functional theory as implemented [13] in CRYSTAL 17 by one of the authors of this 75 study. For Y and Hf atoms, we relied on pseudo-potential (ECP) basis set constructed by Hay76 Wadt and Stevens et al., respectively [14,15] For O atoms, the Durand-Barthelat[16] ECP basis 77 set was chosen in which the $1 \mathrm{~s}^{2}$ core electrons are approximated by a nonrelativistic potential. 80

81

Table 1. Energy Band Gaps (Eg, in eV) of $\mathrm{HfO}_{2}$, $\mathrm{YSH}$ and $\mathrm{YSZ}$, obtained within a plane wave and crystalline orbital calculations.

\begin{tabular}{cccc}
\hline & PBE & PBE0 & B3LYP \\
\hline $\mathrm{c}-\mathrm{HfO}_{2}$ & 4.04 & 6.47 & 6.03 \\
$3.2 \% \mathrm{YSH}$ & 4.38 & 6.77 & 6.26 \\
$6.7 \% \mathrm{YSH}$ & 4.56 & 6.98 & 6.45 \\
$6.7 \% \mathrm{YSZ}$ & 3.58 & 5.83 & 5.39 \\
\hline
\end{tabular}

The relaxed stoichiometric atomistic super-cell structure of c- $\mathrm{HfO}_{2}(2 \times 2 \times 2)$ as well as two cells of YSH of the same size containing 3.2 and $6.7 \mathrm{~mol} \%$ of $\mathrm{Y}_{2} \mathrm{O}_{3}$, (hereafter $(3.2 \%, 6.7 \%$ ) YSH), considered in this report are illustrated in Fig. 1. Our structural results for c- $\mathrm{HfO}_{2}$, also shown in the latter figure, are in good agreement with previously theoretical and experimental outcomes reported by Zhao and Vanderbilt [17] within the local density and generalized gradient approximation and close to the experimental value [18] of $5.08 \AA$ per unit cell $(10.16$ $\AA$ for a $2 \times 2 \times 2$ supercell). According to our knowledge up until now, no experimental or theoretical structural data have been reported for either concentration of YSH.

The energy bandgap values of the above compositions, computed with a pure DFT functional (PBE), and two different hybrid functionals (PBE0, B3LYP), are presented in Table 
92 1. As expected, PBE functional, the least expensive computational method used here in, delivers

93

94

95 considerably smaller bandgaps with respect to the two hybrids PBE0 and B3LYP. A direct comparison of our computed band gap of $\mathrm{c}-\mathrm{HfO}_{2}$ with spectroscopic ellipsometry measurements of $5.8 \mathrm{eV}$ reported by C. Adelmann et al. [19] reveals that the two hybrids, PBE0 and B3LYP, predict considerably improved bandgap energies lying about 0.7 and $0.2 \mathrm{eV}$ above the experiment, respectively. We expect similar method behavior for YSH phases considered in this work.

As far as it concerns the dependence of YSH bandgap on the doping concentration, our computational outcomes, clearly suggest that doping $\mathrm{c}-\mathrm{HfO}_{2}$ with yttrium induces a noticeable bandgap broadening. The observed evolution is in accord with the trend reported by Chen et al [20] who studied the dependence of $\mathrm{Y}_{2} \mathrm{O}_{3}$ concentration on the optical properties of crystalline $\mathrm{HfO}_{2}$ thin films via spectroscopic ellipsometry and concluded to a proportional dependence of the measured band gaps with respect to the doping concentration. Spin-orbitcoupling effects (SOC) on the band structures of pure and doped hafnia using richer basis sets $[21,22,23,24]$. for all atoms have been considered by applying a newly developed scheme in CRYSTAL based on two-component spin-current density functional theory [25,26]. In brief, For Y and Hf we used a fully relativistic effective core potential (ECP) including both the scalarrelativistic AREP and the spin-orbit SOREP parts, developed by Peterson et al. [27] and Figgen et al.[28]. For Y, an associated valence basis set of triple-zeta quality was available for solid-state calculations reported by Laun et al.[29] In the case of Hf, the basis set applied was derived from the associated correlation-consistent double-zeta set for molecular calculations provided by Peterson et al.[24] after we uncontracted the set and removed Gaussians with exponents smaller than 0.1 a.u to avoid linear dependencies. Our final uncontracted Hf valence 
115 basis set is available at CRYSTAL basis set library [30] For $\mathrm{O}$ we used the basis set from 116 Heifets et al. [31]

117 The computations, carried out on the optimized supercells of Fig. 1, exposed that SOC brings 118 minor corrections on the band gaps of all systems considered. Specifically, for pure c- $\mathrm{HfO}_{2}$ 119 SOC increases the respective band gap by $0.004 \mathrm{eV}$ while in the case of $3.2 \%$ and $6.7 \%$ YSH 120 a bandgap opening by 0.007 and $0.006 \mathrm{eV}$ is delivered, respectively. Furthermore, as it can be 121 easily seen in Fig. 2, where we depict the total (DOS) and projected (PDOS) density of states 122 of 3.2\% YSH, the weak spin-orbit-coupling effects evidenced in the band splitting is transferred 123 also on the shape and intensity of the computed DOS and PDOS spectra. 

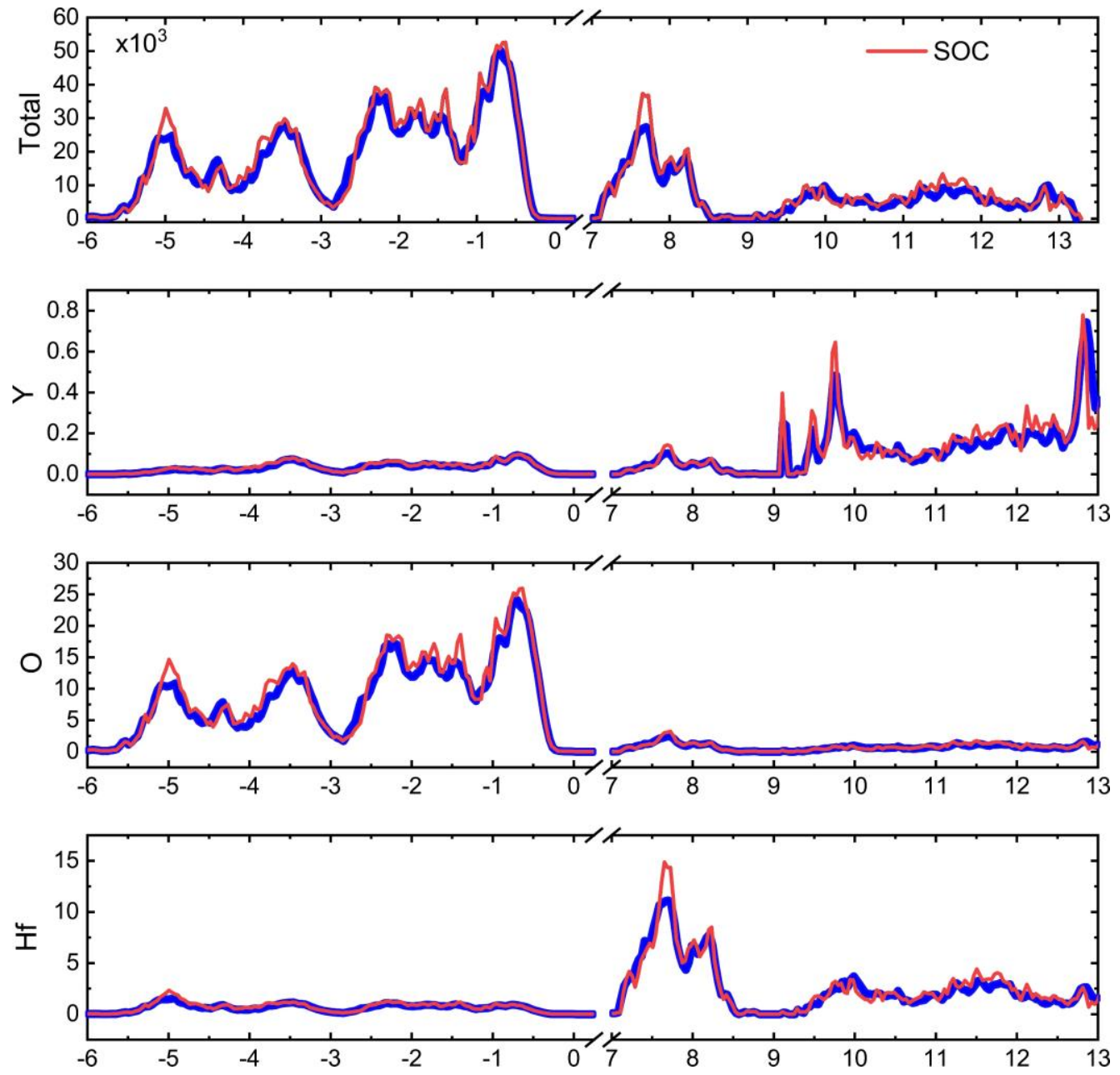

125 126

127

128

129

130

131

132

133

134

135

136
Fig. 2. Total and projected densities of states for 3.2\% YSH obtained at the PBE0. Red colored (P)DOSS spectra correspond to computations considering spin-orbit-couplings (SOC). 
137

138

139

140

Table 2. Electronic contribution to the dielectric $\epsilon_{x x}, \epsilon_{y y=z z}$ components, to the third order susceptibilities $\chi_{x x x x}^{(3)}, \chi_{y y y y=z z z z}^{(3)}, \chi_{y y z z}^{(3)} \operatorname{components}\left(\times 10^{-21} \mathrm{~m}^{2} \mathrm{~V}^{-2}\right)$, of $\mathrm{HfO}_{2}$, YSH 3.2\% and 6.7\% mol.in $\mathrm{Y}_{2} \mathrm{O}_{3}$ and $\mathrm{YSZ} 6.7 \%$ mol. in $\mathrm{Y}_{2} \mathrm{O}_{3}$. In this case $x$, $\mathrm{y}$ and $z$ are placed parallel to the lattice constants $\mathrm{a}, \mathrm{b}$ and $\mathrm{c}$, respectively.

$$
\epsilon_{x x}=\epsilon_{y y}=\epsilon_{z z} \quad \chi_{x x x x}^{(3)}=\chi_{y y y y}^{(3)}=\chi_{z z z z}^{(3)} \quad \chi_{y y z z}^{(3)}
$$

\begin{tabular}{|c|c|c|c|c|c|c|}
\hline \multirow{3}{*}{ c- $\mathrm{HfO}_{2}$} & PBE0 & \multicolumn{2}{|c|}{3.8} & \multicolumn{2}{|c|}{0.9} & \multirow{2}{*}{$\begin{array}{l}0.7 \\
0.8\end{array}$} \\
\hline & \multirow[t]{2}{*}{ B3LYP } & \multicolumn{2}{|c|}{3.9} & \multicolumn{2}{|c|}{1.1} & \\
\hline & & $\epsilon_{x x}=\epsilon_{y y}$ & $\epsilon_{z z}$ & $\chi_{x x x x}^{(3)}$ & $\chi_{y y y y}^{(3)}=\chi_{z z z z}^{(3)}$ & $\chi_{y y z z}^{(3)}$ \\
\hline \multirow{2}{*}{$3.2 \% \mathrm{YSH}$} & PBE0 & 3.6 & 3.7 & 0.7 & 0.8 & 0.6 \\
\hline & B3LYP & 3.7 & 3.8 & 0.9 & 0.9 & 0.7 \\
\hline \multirow{2}{*}{$6.7 \% \mathrm{YSH}$} & PBE0 & 3.5 & 3.7 & 0.6 & 0.7 & 0.6 \\
\hline & B3LYP & 3.6 & 3.8 & 0.8 & 0.9 & 0.6 \\
\hline \multirow{2}{*}{$6.7 \%$ YSZ } & PBE0 & 4.1 & 4.14 & 1.2 & 1.2 & 1.0 \\
\hline & B3LYP & 4.2 & 4.25 & 1.5 & 1.4 & 1.2 \\
\hline
\end{tabular}

141

142

143

144

145

146

147

148

149

150
Let us now turn our attention to Table 2 that summarizes our analytical CPKS outcomes of the dielectric components and the electronic part of the third order NLO susceptibilities of undoped hafnia, 3.2\%, 6.7\% YSH and 6.7\% YSZ. Starting from the relative dielectric tensorial components we see that yttrium doping delivers a slight anisotropy with respect to the perfect cubic phase. This trend is also followed by the computed third-order susceptibilities. Moving now to the properties of interest we see that for all systems both functionals yield third-order susceptibilities not far from the limit of $10^{-21} \mathrm{~m}^{2} \mathrm{~V}^{-2}$ which is considered adequate for certain photonic applications [5]. In addition, it becomes evident that yttrium doping delivers a slight decrease in $\chi_{y y y y}^{(3)}, \chi_{z z z z}^{(3)}$ and $\chi_{y y z z}^{(3)}$ components, a result that is in accord with the evident 
151 bandgap widening occurring upon $\mathrm{Y}$ doping due to the inverse proportionality of $\chi^{(3)}$ to the 152 bandgap of a given material. If we now directly compare the third order nonlinearities of $1536.7 \% \mathrm{YSH}$ to its zirconia analogue, that in this case shares the same local atomistic structure, it 154 is revealed that both lattices feature $\chi^{(3)}$ values of a similar order of magnitudes with the latter 155 being about two times more hyperpolarizable than the former. The revealed ordering is in 156 accord with the wider band gap values of YSH. Furthermore, to facilitate the comparison with 157 previous, and more importantly, future experimental measurements for similar systems, we 158 have used the tensorial components shown in Table 2 to calculate the effective Kerr coefficient $159 \hat{n}_{2}$ of and as shown by Marcaud et al. [5] The obtained outcome of $0.8 \times 10^{-19} \mathrm{~m}^{2} \mathrm{~W}^{-1}$ for $6.7 \% \mathrm{YSH}$ 160 at B3LYP level, lies about 1.6 times lower than the effective Kerr coefficient of $6.7 \%$ YSZ 161 obtained at the same level of theory.

162 Bearing in mind that the nonlinearities discussed so far correspond to the electronic part of $163 \chi^{(3)}$, one should regard the nonlinear coefficients listed in Table 2 as the lowest limit at each 164 level of theory. Based on previous results for YSZ, we expect that the actual NLO response of 165 YSH should be even stronger owed to a combination of effects related to the frequencies of the 166 applied dynamic or oscillating electric fields (c.c. frequency dispersion), possible thermal 167 lattice expansions, and more importantly to vibrational contributions.[32] To address the latter 168 effects we relied on the variation of the dipole polarizability of each unit cell with respect to 169 the atomic displacement along the axial supercell directions. For this task, we applied the 170 formulation corresponding to one of the most common NLO processes, namely, the intensity171 dependent refractive index (IDRI). In this process and by means of the so-called infinite172 frequency approximation, [33] the harmonic diagonal vibronic contribution is simplified as: 
In Eq. (2) $\mathrm{N}$ is the number of atomic centers comprised in the unit cell and $\frac{\vartheta \alpha_{x x, y y, z z}^{e}}{\vartheta Q_{k}}$ the derivative of the axial dipole polarizability component per unit cell with respect to the harmonic normal $Q_{k}$-mode with $\omega_{k}$-frequency. The obtained analytical outcomes within the CPKS

177 approximation are presented in Table 3 and reveal that for pristine hafnia the vibrational 178 contributions are extremely weak with respect to the electronic ones. In striking contrast, our 179 computations at the PBE0 level expose a strong vibrational contribution of $3.0 \times 10^{5}$ and $3.2 \times 10^{5}$ 180 a.u. along the $x$-axis of the unit cells of $3.2 \% \mathrm{YSH}$ and $6.7 \% \mathrm{YSH}$, respectively. As a result, a $181 \gamma_{x x x x}^{\text {tot }}$ value of $9.5 \times 10^{5}$ a.u. which lies about 1.5 times higher than the corresponding $\gamma_{x x x x}^{e}$ value 182 of $3.2 \% \mathrm{YSH}$, has been obtained. A similar behavior is observed for $6.7 \% \mathrm{YSH}$.

183 Taking into consideration that the computed RAMAN intensities are proportional to the 184 square of the first derivative of the dipole polarizability with respect to the corresponding 185 displacement mode, the revealed vibrational effects can be rationalized up to a great extent by 186 a careful analysis of the simulated RAMAN spectra shown in Figure 3. Thereby, we see that 187 the spectrum of pure cubic hafnia is characterized by a single strong absorption band at $640 \mathrm{~cm}^{-}$ 188 1. Despite its large intensity, the respective absorption band lies too high in energy to induce 189 strong vibrational contributions to the second dipole hyperpolarizability along any Cartesian 190 cell direction. On the other hand, in the RAMAN spectra of the two YSH phases one can easily 191 spot several soft active absorption bands appearing in low energies. Their origin should be 192 attributed to the weak but evident deviation from the cubic symmetry of the doped YSH 193 supercell due to the replacement of two 
194 Table 3. Unit cell electronic $\left(\gamma^{e}\right)$, vibrational $\left(\gamma^{v i b}\right)$, and total $\left(\gamma^{\text {tot }}=\gamma^{e}+\gamma^{v i b}\right)$ second dipole 195 hyperpolarizability axial tensorial components of $\mathrm{c}-\mathrm{HfO}_{2}$ and $\mathrm{YSH}$, respectively, computed 196 with the PBE0 functional. All values are given in atomic unit and have been at the PBE0 level 197 of theory applying the same basis sets we used for the geometry optimization.

\begin{tabular}{|c|c|c|c|c|c|c|}
\hline \multirow{3}{*}{$\mathrm{c}-\mathrm{HfO}_{2}$} & $\gamma_{x x x x}^{e}$ & & $\gamma_{x x x x}^{v i b}$ & \multirow{3}{*}{$\sim 0$} & $\gamma_{x x x x}^{t o t}$ & \multirow{3}{*}{$7.9 \times 10^{5}$} \\
\hline & $\gamma_{y y y y}^{e}$ & $7.9 \times 10^{5}$ & $\gamma_{y y y y}^{v i b}$ & & $\gamma_{y y y y}^{\text {tot }}$ & \\
\hline & $\gamma_{z z z Z}^{e}$ & & $\gamma_{z z z z}^{v i b}$ & & $\gamma_{z z z z}^{t o t}$ & \\
\hline \multirow{2}{*}{$3.2 \% \mathrm{YSH}$} & $\gamma_{x x x x}^{e}$ & $6.5 \times 10^{5}$ & $\gamma_{x x x x}^{v i b}$ & $3.0 \times 10^{5}$ & $\gamma_{x x x x}^{t o t}$ & $9.5 \times 10^{5}$ \\
\hline & $\gamma_{y y y y}^{e}, \gamma_{z z z z}^{e}$ & $7.1 \times 10^{5}$ & $\gamma_{y y y y}^{v i b}, \gamma_{z z z z}^{v i b}$ & $0.2 \times 10^{5}$ & $\gamma_{y y y y}^{t o t}, \gamma_{z z z z}^{t o t}$ & $7.3 \times 10^{5}$ \\
\hline \multirow{2}{*}{$6.7 \% \mathrm{YSH}$} & $\gamma_{x x x x}^{e}$ & $5.8 \times 10^{5}$ & $\gamma_{x x x x}^{v i b}$ & $3.2 \times 10^{5}$ & $\gamma_{x x x x}^{t o t}$ & $9.0 \times 10^{5}$ \\
\hline & $\gamma_{y y y y}^{e}, \gamma_{z z z z}^{e}$ & $6.5 \times 10^{5}$ & $\gamma_{y y y y}^{v i b}, \gamma_{z z z z}^{v i b}$ & $0.3 \times 10^{5}$ & $\gamma_{y y y y}^{t o t}, \gamma_{z z z z}^{t o t}$ & $6.8 \times 10^{5}$ \\
\hline
\end{tabular}

198

199 Hf atoms with Yttria and the creation of an oxygen vacancy. This structural distortion,

200 clearly expressed by an elongated lattice constant a (see Fig. 1), activates several soft RAMAN

201 active absorption bands that potentially could contribute in an additive manner to the supercell's

202 second hyperpolarizabilities. In the present case, the vibrational contributions appear

203 considerably strong in the direction of the most anisotropic tensorial component $\gamma_{x x x x}^{e}\left(\neq \gamma_{y y y y}^{e} \cong\right.$

$204 \gamma_{z z z Z}^{e}$ ) which, in turn, corresponds to the most distorted lattice constant a (note that in the current

205 case lattice constants $\mathbf{a}, \mathbf{b}$ and $\mathbf{c}$ coincide with the $x, y$ and $z$ axes, respectively). Indeed, as seen

206 by the values listed in Table 3, $\gamma_{x x x x}^{v i b}$ appears about fifteen and ten times greater than $\gamma_{y y y y}^{v i b}$ and

$207 \gamma_{z z z z}^{v i b}$ or either $3.2 \%$ or $6.7 \%$ YSH, respectively. Finally, as seen by the displacement modes

208 depicted in Fig. 3 the low frequency acoustic modes which play a central role in the respective 

contributes up one third $\left(\sim 1.0 \times 10^{5} \mathrm{au}\right)$ of the overall $\gamma_{x x x x}^{v i b}$ value.
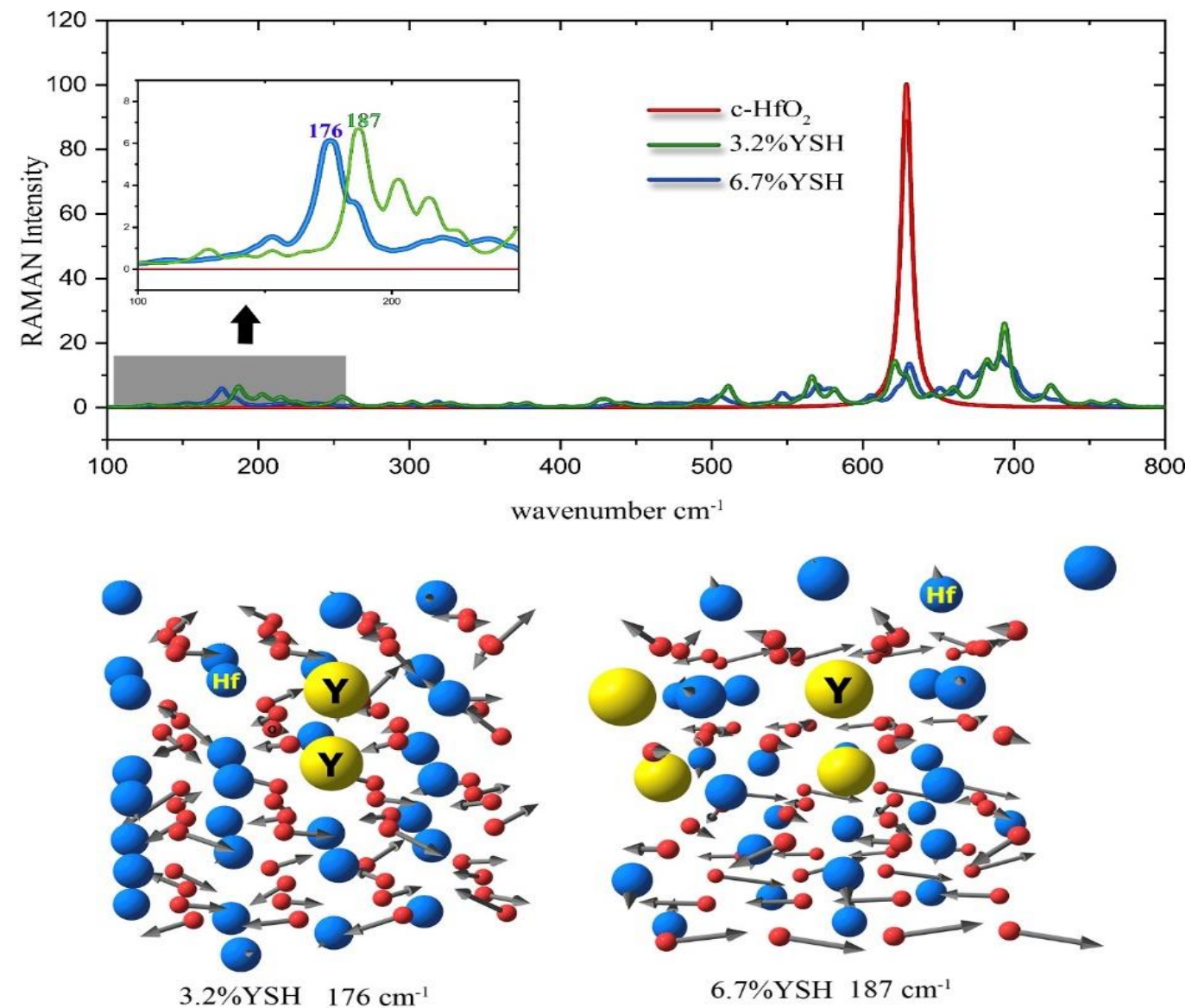

$6.7 \%$ YSH $187 \mathrm{~cm}^{-1}$

Fig.3. RAMAN spectra of 3.2\% YSH and 6.7\% YSH (right) and computed displacement vectors of soft vibrational modes contributing the most on the vibrational supercell hyperpolarizabilities. All computations have been permed at the PBE0 level of theory.

\section{Conclusion}

In this work, the structural, electronic, and optical properties of cubic $\mathrm{HfO}_{2}$ and $\mathrm{YSH}$ bulk have been investigated by first-principles calculations. Our computational data suggest that the NLO capacity of YSH, should be comparable to YSZ recently studied both experimentally and 
implemented in CRYSTAL17, it has been exposed that the electronic properties of YSH do not constitute subjects of strong spin orbit coupling effects. Finally, strong evidence of significant vibrational contributions to the optical nonlinearities of the systems have been found. An article addressing in more details these effects is in preparation.

\section{Acknowledgments}

Part of this work was granted access to the HPC resources of [CCRT/CINES/IDRIS] under the allocations 2019-2020 2020-2021 [A0040807031] made by GENCI (Grand Equipement National de Calcul Intensif). We also acknowledge the "Direction du Numérique" of the "Université de Pau et des Pays de l'Adour" and the Mésocentre de Calcul Intensif Aquitain (MCIA) for the computing facilities provided. H. Denawi thanks ANR project FOIST. CSP acknowledges DST-India for INSPIRE Faculty Fellowship with award number IFA-18 PH217. JKD is grateful to the national science and engineering research council (NSERC) for a postdoctoral fellowship No. 545643. The authors declare that they have no known competing financial interest.

\section{References}

1. A. B. Miller, "Are optical transistors the logical next step?," Nature Photon 4, 3-5 (2010) 2. S. M. Hendrickson, A. C. Foster, R. M. Camacho, and B. D. Clader, "Integrated nonlinear photonics: emerging applications and ongoing challenges," J. Opt. Soc. Am. B 31, 3193 (2014).

3. R. W. Boyd, Nonlinear Optics (Academic Press, San Diego, CA, 1992)

4. M. G. Kuzyk, "Connecting at the speed of light," IEEE Circuits and Devices Magazine 19, $8-17$ (2003).

5. G. Marcaud, S. Serna, P. Karamanis, C. Alonso-Ramos, X. Le Roux, M. Berciano, T. Maroutian, G. Agnus, P. Aubert, A. Jollivet, A. Ruiz-Caridad, L. Largeau, N. Isac, E. Cassan, S. Matzen, N. Dubreuil, M. Rérat, P. Lecoeur, and L. Vivien, "Third-order nonlinear optical susceptibility of crystalline oxide yttria-stabilized zirconia," Photon. Res. 8, 110 (2020). 6. S. K. Pandey, O. P. Thakur, R. Raman, A. Goyal, and A. Gupta, "Structural and optical properties of YSZ thin films grown by PLD technique," Applied Surface Science 257, 6833$6836(2011)$.

7. D.J. Moss, R. Morandotti, A.L. Gaeta, and M. Lipson, "New CMOS-compatible platforms based on silicon nitride and Hydex for nonlinear optics," Nature Photon 7, 597 (2013). 
8. J. Robertson, "High dielectric constant gate oxides for metal oxide Si transistors," Rep. Prog. Phys. 69, 327-396 (2006).

9. O. Ohtaka, H. Fukui, T. Kunisada, T. Fujisawa, K. Funakoshi, W. Utsumi, T. Irifune, K. Kuroda, and T. Kikegawa, "Phase Relations and Volume Changes of Hafnia under High Pressure and High Temperature," Journal of the American Ceramic Society 84, 1369-1373 (2004).

10 J. H. Choi, Y. Mao, and J. P. Chang, "Development of hafnium based high-k materials-A review," Materials Science and Engineering: R: Reports 72, 97-136 (2011)..

11. R. Dovesi, A. Erba, R. Orlando, C.M. Zicovich-Wilson, B. Civalleri, L. Maschio, M. Rérat, S. Casassa, J. Baima, S. Salustro, and B. Kirtman, WIREs Computational Molecular Science 8, e1360 (2018).

12. H. J. Monkhorst and J. D. Pack, "Special points for Brillouin-zone integrations," Phys. Rev. B 13, 5188-5192 (1976)

13. M. Ferrero, M. Rérat, B. Kirtman, and R. Dovesi, "Calculation of first and second static hyperpolarizabilities of one- to three-dimensional periodic compounds. Implementation in the CRYSTAL code.," J. Chem. Phys. 129, 244110 (2008).

14. P. J. Hay and W. R. Wadt, "Ab initio effective core potentials for molecular calculations. Potentials for the transition metal atoms Sc to Hg," J. Chem. Phys. 82, 270-283 (1985). 15. W. J. Stevens, M. Krauss, H. Basch, and P. G. Jasien, "Relativistic compact effective potentials and efficient, shared-exponent basis sets for the third-, fourth-, and fifth-row atoms," Can. J. Chem. 70, 612-630 (1992).

16. J. C. Barthelat, P. Durand, and A. Serafini, "Non-empirical pseudopotentials for molecular calculations," Molecular Physics 33, 159-180 (1977).

17. 1. X. Zhao and D. Vanderbilt, "First-principles study of structural, vibrational, and lattice dielectric properties of hafnium oxide," Phys. Rev. B 65, 233106 (2002).

18. J. Wang, H. P. Li, and R. Stevens, "Hafnia and hafnia-toughened ceramics," J Mater Sci 27, 5397-5430 (1992).

19. C. Adelmann, V. Sriramkumar, S. Van Elshocht, P. Lehnen, T. Conard, and S. De Gendt, "Dielectric properties of dysprosium- and scandium-doped hafnium dioxide thin films," Appl. Phys. Lett. 91, 162902 (2007).

20. X. Chen, L. Song, L. You, and L. Zhao, "Incorporation effect of Y2O3 on the structure and optical properties of HfO2 thin films," Applied Surface Science 271, 248-252 (2013). 21. K. A. Peterson, D. Figgen, M. Dolg, and H. Stoll, "Energy-consistent relativistic pseudopotentials and correlation consistent basis sets for the 4d elements Y-Pd," J. Chem. Phys. 126, 124101 (2007).

22. D. Figgen, K. A. Peterson, M. Dolg, and H. Stoll, "Energy-consistent pseudopotentials and correlation consistent basis sets for the 5d elements Hf-Pt," J. Chem. Phys. 130, 164108 (2009).

23. J. Laun, D. Vilela Oliveira, and T. Bredow, "Consistent gaussian basis sets of double- and triple-zeta valence with polarization quality of the fifth period for solid-state calculations," J Comput Chem 39, 1285-1290 (2018).

24. E. Heifets, E. A. Kotomin, A. A. Bagaturyants, and J. Maier, "Thermodynamic stability of non-stoichiometric SrFeO3- $\delta$ : a hybrid DFT study," Phys. Chem. Chem. Phys. 21, 39183931 (2019).

25. J. K. Desmarais, J.-P. Flament, and A. Erba, "Spin-orbit coupling in periodic systems with broken time-reversal symmetry: Formal and computational aspects," Phys. Rev. B 101, 235142 (2020).

26. J. K. Desmarais, S. Komorovsky, J.-P. Flament, and A. Erba, "Spin-orbit coupling from a two-component self-consistent approach. II. Non-collinear density functional theories," J.

Chem. Phys. 154, 204110 (2021). 
27 K. A. Peterson, D. Figgen, M. Dolg, and H. Stoll, "Energy-consistent relativistic pseudopotentials and correlation consistent basis sets for the 4d elements Y-Pd," J. Chem. Phys. 126, 124101 (2007).

28. D. Figgen, K. A. Peterson, M. Dolg, and H. Stoll, "Energy-consistent pseudopotentials and correlation consistent basis sets for the 5d elements Hf-Pt," J. Chem. Phys. 130, 164108 (2009).

29.J. Laun, D. Vilela Oliveira, and T. Bredow, "Consistent gaussian basis sets of double- and triple-zeta valence with polarization quality of the fifth period for solid-state calculations," J Comput Chem 39, 1285-1290 (2018).

30 www.crystal.unito.it/Basis_Sets/hafnium.html

31 E. Heifets, E. A. Kotomin, A. A. Bagaturyants, and J. Maier, "Thermodynamic stability of non-stoichiometric SrFeO3- $\delta$ : a hybrid DFT study," Phys. Chem. Chem. Phys. 21, 39183931 (2019).

32. D. M. Bishop, J. M. Luis, and B. Kirtman, "Vibration and two-photon absorption," The Journal of Chemical Physics 116, 9729-9739 (2002).

33. B. Champagne, É. A. Perpète", J.-M. André, and B. Kirtman, "Analysis of the vibrational static and dynamic second hyperpolarizabilities of polyacetylene chains," Synthetic Metals 85, 1047-1050 (1997). 